\title{
Le « culte des ancêtres » en pays evhé
}

"Ancestor worship" among the Ewe

\section{Albert de Surgy}

\section{CpenEdition}

\section{Journals}

Édition électronique

URL : http://journals.openedition.org/span/205

DOI : $10.4000 /$ span.205

ISSN : 2268-1558

\section{Éditeur}

École pratique des hautes études. Sciences humaines

\section{Édition imprimée}

Date de publication : 1 septembre 1975

Pagination : 105-128

ISSN : 0294-7080

\section{Référence électronique}

Albert de Surgy, "Le «culte des ancêtres » en pays evhé », Systèmes de pensée en Afrique noire [En ligne], 1 | 1975, mis en ligne le 09 juillet 2013, consulté le 14 novembre 2019. URL : http:// journals.openedition.org/span/205 ; DOI : 10.4000/span.205 
LE "CULTE DES ANCETRES" EN PAYS EVHE

par

Albert de SURGY

PROPOS - L'expression "culte des ancetres" devrait normalement être réservée aux pratiques cérênonlelles adressées à la personne des ancêtres eux-mêmes.

Or, cédant a l'imprécision du parler populaire, divers auteurs rangent sous ce titre les cultes rendus sur les trónesautels des ancêtres, cultes quí ne sont pas adressés aux ancêtres eux-memes, mais font partie des divers cultes rendus sur ce qu'ils ont laisse sur terre aprés eux (1).

I'exposé ci-dessous vise avant tout a ecarter une telle confusion en expliquant la veritable nature du culte des trônes.

Il n'est qu'un aperçu jete sur un travail plus vaste, en cours de redaction, concernant les constituants de la personne humaine en pays Evhe, $a$ travers les rites qui les mettent en valeur.

Quelques précisions sur divers points se trouvent d'ores et đéjà incluses dans ma thèse sur "la geomancie et le culte d'Afa en pays Evhe". Toutes precisions utiles en ce qui concerne la relation des "eléments" avec la structure cosmique et microcosmique se trouveront incluses dans un ouvrage en cours d'achèvement sur "la théorie des cing êléments, une logique immemoriale des systèmes vivants".

Avant de dommencer un exposé sur le culte des ancêtres, il est bon de clarifier nos idées en précisant ce que recouvre exactement la notion d'ancêtre.

I - Qu'est-ce qu'un ancêtre ?

Nous devons, de toute évidence, réserver la notiond'ancêtre à ce qui subsiste de la personne d'un défunt après qu'1l ait ete introdút, par les rites de funeratiles, au pays de ses ancêtres.

(1) A I'examen, il apparalt que les cultes de certains defunts, transformes apparemment en héros, bien que désignés par le nom du defunt lui-même, ne concernent que: solt des objets, devenus autels, sur lesquels le defunt a abandonné, de son vivant, plus ou molns d'une puissance spirituelle qu'il possedalt a profuaton soit les esprits d'hommes ou de femmes sacrifies ou derniers survivants d'une population aneantie ou dispersee par les conquérants. 
Selon la croyance la plus répandue, l'esprit du défunt demeure quelques jours après sa mort et après 1 'enterrement en étroit contact avec le cadavre ou ce qu'il en reste. On dit qu'il se trouve à yome, c'est-à-dire dans la tombe. Dans ces conditions, 4, 5, 8 ou 9 jours après l'enterrement (selon les coutumes particulières des clans) on vient notamment lui offrir, sur la tombe, les libations et de la bouillie (rites qualifies de yophopho ou de dzogbaphophol.

Après ce rituel, l'esprit du mort se libère des liens qui le rattachent au cadavre. Il subsiste, errant et insatisfait, dans un monde intermédiaire, que l'on appelle dukponu (le dépotoir) ou vholime (monde de l'ombre). Il tend à revenir fréquenter les lieux qu'il a connus et c'est alors, principalement, qu'il se manifeste sous forme de revenant. Pendant cette période, à chaque repas, les membres de sa famille lui offrent, comme de son vivant, une part (symbolique) de nourriture.

Les funérailles sont faites pour le détacher complètement du monde qu'il a connu et lui faire rejoindre, au delà du fleuve de l'ouest, la place qu'il mérite au pays de ses ancêtres... de sorte que non seulement il y soit lui-même heureux, mais qu'il cesse aussi, et peut-être surtout, de déranger les vivants.

Après les funêrailles, c'est à la sortie du village, ou bien à la sortie de la cour, à moins qu'on ne les ait appelés comme il convient à l'intérieur, qu'on va porter leurs nourritures aux défunts au cours de cérémonies rentrant véritablement désormais dans le culte des ancêtres.

Mais que subsiste-t-il de 1 'homme après la mort, et que subsiste-t-il en particulier de l'homme après les funérailles?

Ceci nous ramène à la conception Evhé de l'être humain que je vais. $m$ 'efforcer de résumer en quelques lignes :

a) Selon certains, l'homme est composé d'un corps et de deux âmes appelées du nom de luvho, terme qui désigne également, en maints endroits, l'ombre d'un corps. 
L'une de ces ames, la $k u$ zuvho, ou "âme de mort",ombre claire ou àme légère, subsiste après la mort et rejoint finalement le pays des ancêtres. Beaucoup l'assimilent au gbogbo. Elle correspond à notre notion d'âme individuelle (1).

La seconde de ces âmes, l'agbe Zuvhö, ou"âme de vie", ombre noire ou âme dense :

- selon les uns disparaît totalement après la mort. Elle semble dès lors assimilée à l'ombre physique.

- selon les autres reste errer ça et là dans le monde de la vie, au dessus ou à proximité immédiate du monde de la vie (2), et peut déranger les vivants.

Le maitre géomancien Dawukpo Nomenyo, de Dzita (Anlo) est même d'avis que c'est elle qui accompagne un bébé dans le monde et se manifeste sous forme d'amedzodzo ou réincarnation d'ancêtre.

Certains subdivisent $k u$ luvho en quatre et agbe luvho en trois.

Les luvho correspondent à la notion Fon des deux $y \grave{e}$ : un yè long, élément fondamental et dernier de l'homme, et un yè court que G. Montilus (pp. 21-23) assimile au dynamisme vital, ou principe de vie physique.

B. Maupoil (pp. 394-95) précise que le yè long ne se matérialise, sous forme d'apparition, que durant un certain temps avant le retour auprès de Mawu et que l'autre $y \dot{e}$ disparait avec le corps dans la tombe.

Au corps et aux deux luvho, il convient d'ajouter le $S e ̀$, ou destin, puissance d'incitation contraignante qui oriente le vie de l'homme.

Une partie du $S \grave{e} n^{\prime}$ est autre qu'une participation au grand Sè défini par Mawu lui-même, qualifiê de Mawuga Sègbo,

(1) G.K.Nukunya, Some underlying beliefs, pp. 125-126.

(2) Telle est notamment l'opinion rapportée par J. Spieth (Die Bwe stämmer La vie spirituelle des Ho- le côté spirituel de 1 'homme) qui écrit que "L'âme de mort accompagne l'homme dans le tombeau d'où elle part ensuite pour le royaume des morts. I'âme de vie, au contraire, quitte le corps humain immédiatement après la mort ; cette âme va ça et là en gémissant et en cherchant une demeure et n'en trouve pas". 
une autre est assimilée au kpoli. Une autre correspond à l'orientation conférée à l'individu par son père et sa mère. Une autre correspond à l'orientation conférée à l'indiviđu par l'ancêtre qui se"réincarne" en lui en qualité d'amedzódzo (notion identique à la notion Fon de djoㅡㅡㅇㅢ).

b) D'autres ne distinguent qu'une seule sorte de Zuvho: c'est le $k u-l u v h o$ ou âme individuelle de précédemment.

Ils distinguent semblablement le $S \grave{e}$ et le corps physique ; mais ils remplacent la notion d'agbe luvho par celle de gbogbo (3) (terme signifiant esprit et souffle) principe actif, énergétique, source de chaleur et de manifestation.

A la mort, le gbogbo d'un individu peut, selon les cas, se transformer en esprit errant et devenir vodu (cas des mauvais morts privés de funérailles) ou intervenir, à titre d'amé-dzodzo dans la formation du corps d'un bébé (on dit qu'il pétrit la glaise dont sera tiré le corps de l'enfant).

Cependant, parallèlement, le gbogbo demeure associé au Zuvho pour constituer le ñoli : $1^{\prime \prime}$ ancêtre "réincarné" n'en demeure pas moins au pays des ancêtres ; il peut se réincarner, ou plutôt se remanifester sans limite de temps ou de nombre dans plusieurs de ses descendants.

De même l'esprit du mauvais mort, pourtant errant, n'en est pas moins parti lui aussi chez Mawu.

Les rites et les croyances qui s'y rattachent montrent, à l'évidence, que le gbogbọ se présente simultanément sous deux aspects $\log$ iquement distincts mais pourtant indissociables.

Il existe un gbogbo compatible avec l'être immuable, en repos, de Mawu, et un autre gbogbo incompatible avec cette paix, agité et tourmenteur, entretenant au contraire le mouvement et I'expression de I'être humain ; celui-ci émanant de celui-là et étant identifié à celui-là dans son principe.

Le souffle ( $g$ bogbo) est, en effet, directement accordé

(3) Nukunya (art. cit., p. 124) assimile la notion de gbogbo a celle d'âme de vie. 
à l'homme par Mawu. Tenant à une telle source, il est inépuisable (d'ou les possibilités de "reincarnations" indéfinies du même ancêtre). Cependant, en tant que quantité d'énergie vitale accordée au plan de la manifestation, mais non intégralement épuisée en manifestation, il subsiste, errant, après la mort, au dessus du plan de la manifestation et devient éventuellement vodu dont il faut constamment entretenir la puissance par des sacrifices sanglants libérateurs d'énergie analogue (notons que, de même, dans les traditions hermétiques, le souffle est jugé issu de l'enveloppe éthérée du monde, laquelle, contenant tout en puissance, correspond à la notion Evhé de Mawu). (1)

Le gbogbog qui s'en retourne a Mawu n'est autre que la présence créatrice immanente de Mawu au sein de toute créature (2). Le $g b o g b o$ susceptible d'errer par le monde après la mort est celui qui correspond au souffle vital, circule avec le sang, et siège principalement dans la tête, elle-même traitée comme un vodu car elle contient ce qui est de la même nature qu'un vodu.

Comme le gbogbog, à sa source, sur la sphère de Mawu, reste pure potentialité, nous le distinguerons du gbogbo actualisé au coeur de la substance vivante en l'écrivant entre parenthèses et en le situant au-dessus de tous les autres principes constitutifs de l'homme.

(Nous remarquerons que cette etroite relation entre le gbogbo de pure potentialité et le gbogbo actualisé est analogue à la localisation de la faculté humaine appelée ayè (faculte de ruse, celle qui permet a l'homme de conserver ses intentions secrètes, affectée par le dynamisme de la potentiali_ té), non seulement dans le ventre, source première de la vie, mais aussi sous forme d'intelligence (nunya ay $\dot{e}$ ), dans la tête, responsable de l'activation du corps vivant.

(1) Poimandrès, 17 : "de l'éther, la nature reçut le souffle vital".

(2) Nukunya (art. cit., p. 124) affirme que le gbogbo est un petit morceau de l'être suprême en l'homme. 
(gbogbo)

\begin{tabular}{|l|}
\hline Ku luvho \\
\hline Agbe zuvho \\
\hline$S e$ \\
\hline $\begin{array}{l}\text { corps } \\
\text { physique }\end{array}$ \\
\hline
\end{tabular}

(a)

\begin{tabular}{|l|}
\hline Luvho \\
\hline Gbogbo \\
\hline$S e ̀$ \\
\hline $\begin{array}{l}\text { corps } \\
\text { physique }\end{array}$ \\
\hline
\end{tabular}

(b)

\begin{tabular}{|c|c|}
\hline \multicolumn{2}{|c|}{$(g b \underline{g} g b \underline{)})$} \\
\hline Daogbemesro & Luvho \\
\hline Ak rama & $G b \underline{g} g b \underline{0}$ \\
\hline Gbetsi & Sè \\
\hline Ombre & $\begin{array}{l}\text { corps } \\
\text { physique }\end{array}$ \\
\hline
\end{tabular}

(c)

Les principes constitutifs de l'homme dans la tradition Evhe

c) Le schéma des constituants de la personne humaine se complique encore du fait qu'à chaque principe est, en outre, associé un principe jumeau, principe exterieur à l'individu, mais pourtant intimement lié à l'individu, ce qui donne à l'ensemble des constituants de la personne humaine, la même structure apparente qu'un afadu.

- I'ombre (íöoli ou agbe luvho) jumelée au corps physique.

- le gbetsi, jumelé au destin. Il s'agit de la promesse faite par l'individu lui-même, avant de quitter la sphère divine, de mener tel ou tel type d'existence. Cette parole donnée, personnifiée, revient rappeler à l'ordre l'individu dès qu'il tend à s'en écarter.

- l'ak zama, ou génie de la chance individuel, esprit gardien jumelé à l'esprit propre de I'homme.

- l'épouse ou l'époux de l'origine (dzogbeme sró), jumelé à 1 'âme individuelle. Il $s^{\prime}$ agit du type d'épouse ou d'époux ldéal que Dieu definit pour tout individu en l'envoyant au monde... sorte d'alter ego dont il recherche la concrétisation a travers l'amour libre ou le mariage. 
catégories dans le détail desquelles nous n'entrerons pas :

Ainsi existe-t-il 4 luvho associes, deux a deux, par paires, et résidant devant et derrière, à droite et à gauche de l'individu.

Trois autres personnages analogues à l'epouse prédestinée : ce sont la tante paternelle ldéale (ñolime tasi), la mère idéale (ñolime nó), l'ami idéal ou la seconde épouse idéale.

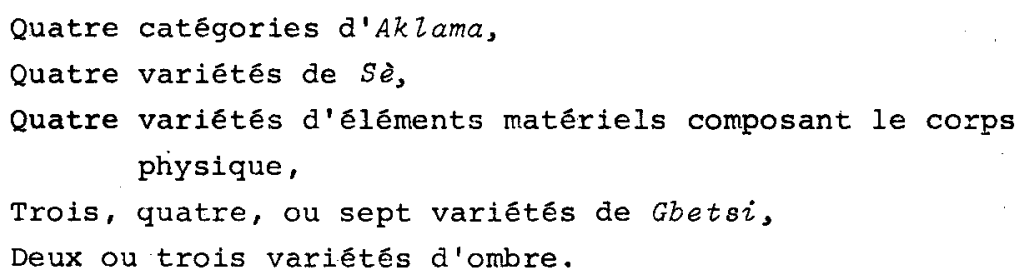

A la mort, l'homme perd tous les principes extérieurs qui lui étaient jumelés. Son corps physique est rapidement détruit et c'est la fin de sa destinée en ce monde.

Le gbogbo vital, en cas de mort survenue avant qu'il n'ait été épuisé, reste éventuellement errer de par le monde.

Ne subsistent plus que le luvho et le gbogbo divin, constituant le ñoli que les funerailles introduisent au pays des ancêtres. Mais, de par son union au $g b \underline{b} g b \underline{o}$, sans lequel il cesserait d'exister, l'âme du défunt demeure indéfiniment capable d'entrer de nouveau en contact avec les êtres vivants.

\section{II - Pratiques relevant du "culte des ancêtres".}

Les vivants demeurent, à vrai dire, en relation permanente avec leurs ancêtres et $n$ 'accomplissent aucun sacrifice important sans, d'une manière quelconque, les y faire participer... de sorte que le "culte des ancêtres" apparait à l'état diffus dans tous les aspects de la religion.

Peut-on d'ailleurs parler valablement de "culte" en ce qui concerne les relations que les vivants entretiennent avec des défunts qui ne cessent jamais d'être prêsents (à leur manière, cela va de soi) à côté d'eux ? En bonne logique, si 
nous devions tenir à l'expression "culte des ancêtres" pour désigner toutes les pratiques qui entretiennent ces relations, il faudrait parallèlement utiliser l'expression "culte des vivants" pour désigner l'ensemble des pratiques par lesquelles les vivants entrent en relation (d'échange) les uns avec les autres.

Il est cepenaiant des circonstances où, en dehors d'une simple pensée à l'intention des ancêtres, d'une simple inspiration reçue des ancêtres, d'une simple association des ancêtres à d'autres activités du culte, on s'adresse exclusivement, et de maniēre institutionnalisée, aux ancêtres pour traiter avec eux et en obtenir des bienfaits analogues à ceux que l'on pourrait obtenir d'une divinité. Je pense que c'est en ce sens, et avec les réserves faites plus haut, que l'on peut parler d'un véritable culte des ancêtres; et je n'en ai jusqu'à présent rencontré que quatre aspects.

\section{a) Cérémonie d'accueil de l'amedzodzo:}

Lorsqu'un ancêtre est revenu dans un enfant sous forme d'amedzodzo, il est rituellement accueilli par la famille qui lui verse en outre des libations et y fait participer la collectivité de tous les autres ancêtres. C'est une cérémonie très simple qui ne comporte pas de sacrifice sanglant. I'ancêtre en question est satisfait d'avoir tout simplement été reconnu.

\section{b) Sacrifice sanglant aux ancêtres.}

- Il est des cas où on sacrifie à un ancêtre déterminé (sacrifice au ñoli de son père ou de sa mère).

- des cas où l'on sacrifie à une catégorie d'ancêtres déterminée (anciens géomanciens, anciens chefs de guerre, anciens prêtres du vodu v...).

- des cas où l'on sacrifie à la totalité des ancêtres du lignage ; le sacrifice est alors appelé aphedoxexe, ce qui signifie "boucher" (au sens de combler, rassasier) le Iignage (mot a mot "le creux de la maison", la maison étant comprise 
comme délimitant une puissance cachée d'où proviennent et vers laquelle reviennent les membres de la famille).

Les rites sont les mêmes dans les trois cas.

Parfois les défunts se contentent d'être servis sur le sol. Dans ce cas on dépose sur le sol de la cour en guise d'autel, une, deux ou trois brassées de feuilles aviayiañgba (provenant de l'arbre newbouldia laevis ou jatropha curcas).

Si les defunts prêfèrent être servis au fond d'un trou, on dépose ces mêmes feuilles au fond du trou et on place encore par dessus une écuelle. On peut, de plus; entourer le trou de tissu blanc. Gênéralement on fait un peu plus loin un second trou, de façon à pouvoir y offrir séparément le sang et la nourriture sacrificielle aux "mauvais morts" qui ne doivent jamais être servis en compagnie des morts ordinaires.

Enfin, les défunts peuvent demander à être servis contre le mur de la case. Dans ce cas, au dessus de 2 ou 3 tas de feuilles aviayiañgba, on épingle, contre ce mur, un linge blanc. Il faut mettre premièrement toutes les offrandes (boisson,sang, nourriture) en contact avec ce linge (cela généralement en deux ou trois points) avant qu'elles ne coulent ou ne tombent en bas sur les feuilles.

Selon les exigences des ancêtres, les cérémonies peuvent débuter et s'achever le même jour, ou débuter le soir, se poursuivre par une veillée, et s'achever le lendemain ou même le surlendemain.

c) Nourriture portée aux ancêtres à l'entrée du cimetière ou a la sortie de la cour.

A Dzolo et dans les villages environnants, il est très fréquent de préparer de la nourriture, sans qu'il y ait eu sacrifice proprement dit, et de la porter aux ancêtres à la sortie du village, près du cimetière du quartier. On appelle cela ñolinudhadha, c'est-à-dire "cuire de la nourriture pour les ñozi". 
C'est après avoir déposé nourriture et boisson à l'endroit approprié, qu'on appelle les ancêtres, qu'on leur explique le pourquoi de la cérémonie, qu'on leur demande éventuellement la paix ou certaines faveurs, et qu'on leur verse des libations. ( Les défunts étant autrefois enterrés dans Jes cases, cette coutume parait identique à celle d'offrir tout simplement de la nourriture aux défunts au bord du chemin, à l'extérieur de la cour).

d) Nourriture portée aux ancêtres évoqués chez une devineresse.

Lorsqu'on se rend chez la devineresse ventriloque xoyoto évoquer les esprits des ancêtres et les faire parler, il est bon de leur apporter également de la boisson et des plats de nourriture que la devineresse leur offre dans son sanctuaire, quand, ayant répondu à son appel, et conversé avec l'auditoire rassemblé dans l'antichambre, ils sont censés y être tous rassemblés.

Cette façon de faire se nomme nudhadha tsoyi xogbo, ce qui signifie "cuire de la nourriture ( $n u d h a d h a)$ pour aller (tsoyi) dans la case $(x \underline{g} g \underline{\underline{O}} \underline{)})$ ".

Elle peut concerner un seul ancêtre ou un ensemble d'ancêtres.

(A Dzolo, elle se trouve toujours jumelée avec un dêpôt préalable de nourriture à l'entrée du cimetière).

III - Le culte des trônes ancestraux (Togbi zikpi).

Le culte adressé aux divers Togbi zikpi (Togbi signifie grand'père ou ancêtre, et zikpi signifie siège, trône) peut être superficiellement compris comme un culte adressé collectivement aux ancêtres d'un lignage, sur un autel en forme de trône a cing pieds, réservé à cet effet.

Ainsi, dans son ouvrage sur la religion traditionnelle des Anlo (1), C.R. Gaba assimile à plusieurs reprises le culte

(1) Anlo traditional religion - Ph. D. Thesis - London University of London, 1965, 495 pp. illus. $101 / 4$. 
des trônes avec le culte des ancêtres.

Il parle (p. 130) de la "représentation symbolique des ancêtres : un petit trône de bois sculpté".

Il présente le lavage de ces trônes, avant tout sacrifice, comme (p. 131) un "lavage des ancêtres" (bathing and anointing the ancestors).

Evoquant le dépot des prémices de la récolte d'ignames sur ces trônes, $i 1$ parle des "prémices offertes aux ancêtres", etc...

Pareille assimilation, assez largement répandue, est très regrettable. Nous allons montrer que si les ancêtres ne sont pas insensibles au culte des trônes qu'ils ont institués ou qu'ils ont détenus durant leur existence, tout comme ils ne sont pas insensibles au respect de tout ce qu'ils ont laissé d'autre en mémoire d'eux-mêmes : coutumes, lieux historiques, divinités... le culte de leurs trônes ne leur est en aucune manière adressé et qu'il ne s'agit là que du culte d'une variété singulière de divinités traditionnelles ( $t r o$ ). Les ancêtres ne sont ni plus ni moins concernés par ce culte-là que par le respect de toute pratique religieuse ou sociale susceptible de faire de leur descendance l'heureuse continuation de ce qu'ils ont été.

a) Conditions d'installation des trônes.

La première constatation à faire est que toutes les familles ne possèdent pas de Togbi zikpi.

or est-il possible que certaines familles soient ainsi empêchées de rendre un culte approprié à leurs ancêtres ?

Des trônes sont institués dans les branches familiales qui n'en possédaient point. Or les conditions dans lesquelles il s'en crée de nouveaux ne peuvent manquer de nous aider à en comprendre la nature.

Il existe en pays Evhé trois catégories de trônes : 
$\left.1^{\circ}\right)$ des trônes remontant à une époque immémoriale (1): ceux des chefs ou rois traditionnels, c'est-à-dire ceux des grandprêtres ou pontifes ; notamment, à proximité du 1ittoral, ceux des prêtres et grand-prêtres de $N y i g b i a$, connus sous le nom de Nyigbla zikpi.

Il ne s'en crée plus.

$\left.2^{\circ}\right)$ des trônes de guerre, appelés Avha zikpi, détenus par des chefs qualifiés de "chefs de guerre" (Avha fia), installés dans une famille a la suite des exploits réalisés sur le charp de bataille par un de ses membres, à la tête d'un groupe de guerriers.

Le terme de chef de guerre peut prêter à confusion car il ne s'agit nullement là de quelqu'un promu à une fonction de haut commandement militaire, mais de quelqu'un consacré chef - et dès lors ne participant plus ni aux combats ni. à la conception des combats -, en raison d'exploits guerriers.

Selon Seke Axovi (2), celui que le peuple désignait pour le diriger au combat se faisait remettre non seulementune machette ou sabre (eyi) de guerre qui lui valait le titre d'eyifia, mais aussi un petit siège à cinq pieds dont il se servait non seulement pour $s^{\prime}$ asseoir au sec, mais pour $y$ appuyer le canon de son fusil dans la position du tireur couché. C'est au retour d'une de ses campagnes victorieuses que son siège était transformé en trône sacré.

Nous voyons par là que le trône était consacré du vivant même de son fondateur; bien avant qu'il ne soit devenu un ancêtre. Après sa mort, cet ancêtre est considéré comme ayant été à l'origine de quelque chose, comme ayant légué quelque chose à ses descendants ; mais ne saurait en aucun cas être confondu avec ce quelque chose. Les prières enregistrées à l'occasion des cérémonies du culte sont très explicitesà ce sujet: Ayant appelé tous les ancêtres de la famille avant un

(1) Contrairement à ce que certains ont laissé entendre, lestrônes à cing pieds ne sont nullement une spécialité Ashanti. Les anciens trônes Evhé sont d'origine Adja et n'ont pas été empruntés aux groupes Akan.

(2) Vieil intrônisateur de chefs, par ailleurs maitre géomancien membre du clan Lafe à Angola. 
sacrifice au trône, le prieur déclare par exemple (1): "Voyez l'eau que j'ai en main.Car en vérité votre ancêtre Akaba a trouvé quelque chose, et ce qu'il a trouvé est le Togbi aikpi qu'il a apporté dans la famille ou nous sommes nés. Vous l'avez adoré jusqu'à nos jours ; nous aussi nous suivons vos pas. Maintenant le Togbi $2 i k p i$ rous revient, les enfants ! Oui, le Togbi zikpi est lavé aujourd'hui. Nous devons sacrifier des bêtes sur lui et il est nécessaire que nous vous avertissions car (jadis) vous avez fait ce travail là".

Il ajoute plus tard : "Tu (ancêtre Akaba) étais le chef du village; et aujourd'hui c'est le jour des cérémonies pour la chose que tu as trouvée".

La veille des cérémonies de première consécration d'un trône, on sacrifiait un bouc pour les ancêtres défunts.

Nous voyons par là que le sacrifice aux ancêtres (nul n'aurait su omettre de les avertir et de les faire participer à un tel évènement) était parfaitement distingué de ce qui était accompli ensuite pour le trône, et qu'accompli, de plus, à un jour distinct. de la semaine (chaque jour de la semaine étant propice à une catégorie distincte d'évènements) il relevait d'une autre catégorie de sacrifices.

Après le sacrifice aux ancêtres, on organisait une veillée pendant laquelle le siège, soustrait à son possesseur, était lavé à l'eau de mer puis à l'eau du zio ou de l'Afram (rivières tranquilles) ou à l'eau de pluie. Un pot de la même eau douce était déposé à côté de lui dans la pièce destinée à lui servir de sanctuaire. Puis on l'enduisait d'une poudre noire fabriquée en calcinant trois têtes d'ennemis ramenées de la guerre par le chef.

Enfin on sacrifiait dessus un bélier. Celui à qui était confiée la tête de ce bélier prenait le titre d'agbotadhua et devait désormais remplacer le chef comme entraineur d'hommes au

(1) Enregistré à Anyako le 15 septembre 1968 dans la famille Akaba. 
combat.

Il est significatif de constater que la réussite guerrière, au lieu de promouvoir l'officier en tant que guerrier, le retirait au contraire du métier des armes pour en faire essentiellement un homme consacré.

Il arrivait aussi que des guerriers victorieux capturent des trônes ennemis.

Ces trônes pouvaient être consacrés au retour de campagne selon un rite analogue.

Mais ils pouvaient être tout simplement entreposés, comme des trophées, dans une pièce de la maison de celui qui avait dirigé les guerriers. Des années ou des dizaines d'années plus tard, à la suite de malheurs répétés, d'anomalies ou de prodiges, un devin pouvait apprendre au chef de famille que ce butin était dangereux, que c'est lui qui "dérangeait les gens" et qu'il convenait de lui rendre un culte (1), Il était ainsi promu au rang de tro.

Là encore, comment confondre ce culte nouvellement institué avec un culte des ancêtres ?

$\left.3^{\circ}\right)$ une troisième catégorie de trônes est celle des "trônes de richesse" ou Hozikpi, installés dans la famille de personnes ayant été suffisamment riches pour s'acheter un ou plusieurs esclaves.

Ces trônes peuvent, de même, être installés du vivant de ceux qui ont acheté des esclaves, ou après leur mort.

On leur entoure le pied central d'autant d'anneaux de cauris que d'esclaves ayant été acquis par la famille.

Ils sont considérés comme ayant été eux aussi légués par le chef de famille acquéreur d'esclaves. Ainsi dira-t-on:

(1) Ce n'est qu'en 1966 qu'un trône à cinq pieds, ancien butin de guerre, fut consacré comme Avha zikpi dans la famille Tsiamé de Dzolo (Cir. de Keve, Togo). 
"Le $a i k p i$ de Togbi Eto est venu embrasser le cou de son petit fils" (1). Ou bien "Le zikpi du Togbi zewu (ancêtre fondateur du trône) a dit que..." (2).

Il est, en outre, précisé dans une prière : "Togbi Zewu, après la mort de celui que tu avais acheté, cette personne est devenue un tro... Ton $z i k p i$ a dit que c'était grâce à lui qu'Edo se trouvait guéri. Ton zikpi a demandé à Edo de le remercier avec un bélier, un coq et des boissons...(2)

Nous venons de voir rituellement précisé ce que nous ont expliqué par ailleurs plusieurs informateurs : à savoir que le Hozikpi est l'autel d'un tro constitué par l'esprit de l'esclave défunt n'ayant pu être introduit au pays de ses ancêtres par des funérailles coutumières appropriées, condamné donc à errer de ci de là en dérangeant plus particulièrement les gens de la cour où il a vécu.

Et, de même, l'Avha Zikpi sert d'autel à l'esprit des guerriers frappés de mort violente au cours des combats. Le culte rendu sur le trône de guerre ne s'adresse nullement à l'ancêtre fondateur du trône et à ses descendants, mais à des esprits errants dont il a été responsable de la mauvaise fortune. C'est pourquoi il peutêtre institué du vivant même de I'ancêtre fondateur.

(Quant aux trônes de Nyigbla, mon hypothèse - ce n'est là qu'une hypothèse - est qu'il servait d'autel à l'esprit des prêtres-rois de la tribu, rituellement sacrifies après sept ans de règne et dont la collectivité des autres prêtres était responsable de la mort.)

b) Sacrifices aux trônes.

L'étude, mêrue superficielle, des sacrifices aux trônes accuse la différence radicale qui les sépare des sacrifices aux ancêtres.

(1) Enregistrement effectué à Dzolo, le 18 décembre 1968, lors d'un sacrifice à Ho aikpi.

(2) Enregistrement effectué à Dzolo, lors d'un sacrifice, le 12 avril 1970 . 
Tout d'abord il est fréquent que le sacrifice au trône soit précédé d'un sacrifice aux ancêtres. Ce sacrifice aux ancêtres a généralement lieu la veille, donc un autre jour de la semaine, avant la veillée qui précède le sacrifice au trône.

Il a généralement lieu au portail ou dans la cour, et non au sanctuaire du trône.

Il est de préférence exécuté par le chef de lignage ou un représentant du chef de lignage qualifié à cette occasion de tsiphodhito, personnage parfaitement distinct du prêtre du trône appelé zikpinunola (c'est-à-dire "celui qui reste à la bouche du trône").

Non seulement un sacrifice aux ancêtres prêcède un sacrifice aux trônes, mais après avoir sacrifié au trône lui-même, avant de consommer la nourriture sacrificielle et d'en offrir une part au trône, on se rend en offrir une part aux ancêtres au même endroit où on leur a éventuellement offert, la veille, leur propre part de nourriture. (A Dzolo et aux environs, cette part leur est portée à l'entrée du cimetière). Telle est la coutume, aussi bien en ce qui concerne les trônes de richesse que les trônes de guerre.

Lors d'un sacrifice observe à Dzolo le 26 janvier 1970, le sacrificateur ayant appelé le trône du nom de l'ancêtre qui l'avait apporté au village, m'expliqua, sans la moindre ambiguité, le lendemain, qu'à cette occasion "on prie le trône de guerre et non les ñoli (défunts). Les ñoli ne sont pas sur le trône".

Agbevina Dovhu, intronisateur des chefs de Dzolo, me précisa à son tour : "Il est question des ancêtres dans le culte des 2ikpi. Mais on les appelle et on leur présente de la nourriture uniquement parce qu'ils ont légué le zikpi à leurs descendants. Le zikpi est là en leur mémoire, mais le culte du zikpi ne s'adresse pas à eux".

Emedodzi Alonyo, chef de la famille Tsiamé du même village me confirma que "ce n'est pas aux ñoli qu'on s'adresse quand on prie le zikpi. Les $\bar{n} \underline{\underline{o}} \mathrm{l} i$ ne sont pas sur le zikpi. Le 
zikpi est comme un trọ (divinité) légué par les ancêtres".

Dawukpo Noményo, de Dzita (Anlo), prêtre des ancêtres de sa famille, est lui aussi d'avis qu'"il n'y a rien des ancêtres eux-mêmes sur un $2 i k p i$. On appelle les ancêtres à côté".

c) Rites destinés à détacher l'esprit d'un défunt du trône qu'il a détenu de son vivant.

Loin que l'esprit du défunt soit mis en relation, après sa mort, avec le trône qu'il a fondé ou détenu, on cherche, au contraire, par un rite de renvoi, à détacher son esprit de ce trône.

Ce rite a lieu à l'occasion des funérailles du chef défunt qui sont, parallèlement, l'occasion d'intrôniser son successeur.

On confectionne à cet effet un trône à cinq pieds miniature qui servira de substitut, pour le défunt, au véritable trơne qu'il doit céder. Et, après l'avoir convenablement lavê, on va le déposer en brousse à l'intérieur d'une petite construction de paille où il sera totalement abandonné.

Avant de s'en retourner, on prie en versant des libations d'eau enfarinée et d'alcool, disant, par exemple :

(Anloga, selon l'intrônisateur seke Axovi) : "Nous t'arrachons dès aujourd'hui du Tegbi zikpi. Dès aujourd'hui le Togbi Zikpi ne t'appartient plus"!

(Edzi, selon l'intrônisateur Kubi Anlovo) : "x... (nom du défunt), voici maintenant ton trône (le miniature). Ne viens plus sur le trône de chez nous. Aujourd'hui, nous enlevons ta main de dessus le Togbi 2ikpi".

"... Voici pour boire, ne viens plus boire avec nous !"

C'est ensuite seulement qu'on lave le trône sacré, qu'on le met éventuellement en contact avec le nouveau chef et qu'on lui sacrifie un bélier.

Dans ces conditions, l'esprit du défunt ne restera pas 
connecté au trône, que ce soit pour communiquer quelque chose de lui-même à son successeur ou que ce soit pour bénéficier d'un apport périodique d'énergie spirituelle. Cela l'empêcherait de rejoindre le pays des ancêtres et le retiendrait indûment dans le monde de la vie.

D'une part, c'est du pays des ancêtres que le défunt veillera sur toute sa familie; d'autre part, c'est grâce aux onctions et aux sacrifices sanglants dont il sera l'objet que le trône conservera l'énergie spirituelle - en définitive accordée par Mawu lui-même -, nécessaire à la satisfaction de son détenteur et de sa famille.

Pour nous résumer, constatons que toutes les familles ne disposent pas de trône; que les trônes sont desservis par des prêtres différents de ceux du culte des ancêtres ; qu'on ne leur sacrifie pas le même jour qu'aux ancêtres; qu'à l'occasion des sacrifices aux trônes on va présenter ailleurs aux ancêtres une part de nourriture sacrificielle; qu'ils sont souvent installés du vivant même de l'ancêtre fondateur; que lors des funérailles d'un chef, loin de 1'y connecter, on arrache au contraire son esprit du trône pour qu'il puisse rejoindre aisément le pays des ancêtres.

Il n'en faut pas plus pour démontrer que le culte des trônes ne saurait appartenir à la même catégorie qu'un authentique culte des ancêtres.

\section{IV - Nature du culte des trônes.}

La nature du culte rendu sur les trônes des ancêtres est éclairée par l'examen de quelques autres cultes faisant intervenir de petits trônes miniatures à cinq pieds.

Tatrọe: (le petit tro de la tête, ou la tête en tant que petit $\left.t^{\prime} \underline{o}\right) ; \quad l$ 'une des quatre variétés de tête (1), s'installe à quelqu'un, sur la recommandation d'Afa, sous forme d'un petit trône à cinq pieds auquel est associé un couple de statuettes taillées dans le même bois. Ces statuettes représentent les

(1) La tête ordinaire, ou agbeta, Tagba, Tatrọe et Tase. 
jumeaux (venavi); l'une d'ellesest mâle et porte habituellement la main à sa tôte; l'autre est femelle et conserve les bras ballants.

Aǩlama, le gênie individuel de la chance peut également être installé à quelqu'un sur la recommandation d'Afa. Les modalités d'installation varient quelque peu selon que le sujet à qui l'on installe Aklama est un cultivateur, un marchand, un chasseur ou pêcheur, ou un géomancien. Quoi qu'il en soit, cette installation fait toujours intervenir un petit trône à cinq pieds et deux statuettes jumelles.

Adekpoe (le petit enclos de la chasse) est un petit sanctuaire délimité par une cloture, installé dans la cour des chasseurs qui ont tué de gros animaux. Il est ensuite hérité par les descendants de ces chasseurs, qu'ils soient ou non,euxmêmes, chasseurs.

Lorsqu'un chasseur vient de tuer, pour la première fois, un animal à l'esprit puissant (léopard, rhinocéros, buffle... ou l'oiseau noir dzogbelosu), il doit placer dans sa bouche une herbe des jumeaux (venavigbe) et ne plus parler à personne.ceux qui savent ce que la chose veut dire s'empressent en le voyant de faire appel aux chasseurs voisins confirmés, de façon qu'ils l'accompagnent jusqu'au cadavre de l'animal et procèdent sur Iui à des cérémonies d'apaisement et de libération de son esprit, avant même de le ramener au village. Alors seulement, le nouveau chasseur se laisse ôter de sa bouche l'herbe qu'il morảait et peut enfin parler. à ses semblables pour leur raconter son exploit.

S'il ne dispose pas déjà chez lui d'un adekpóe, il lui faudra en faire édifier un.

A l'intérieur d'une grande écuelle que l'on dépose au milieu de la clôture, on dépose des herbes magiques, un petit fusil miniature, la tête de l'animal, ainsi que la partie blessée du corps de l'animal et la balle qui l'a tue. Par la suite, le chasseur devra $y$ ajouter toutes les têtes ou seulement les mâchoires inférieures et quelques os des gros animaux qu'il tuera, et parfois encore les têtes des boucs qu'il fera sacrifier sur tout cela. 
Enfin, à côtế de l'écuelle, on aépose un petit trône a cinq pieds accompagné de deux statuettes jumelles, l'une mâle, l'autre femelle.

A l'entrée de l'enclos, on dépose enfin généralement une pierre trouvée dans une rivière et une pierre rouge latéritique.

Lors d'un sacrifice, du sang est versé aussi bien dans le contenu de l'écuelle que sur le petit trône et les statuettes, et sur les plerres.

Qu'a-t-on constitué là ? Un autel pour entrer en relation avec les esprits ( $g b \underline{g} g b o$ ) des gros animaux que le chasseur a fait perir de mort violente et qui, devenus errants, pourraient se reporter contre le responsable de leur mort. Au contraire, entretenus convenablement par des incantations, les onctions magiques et les sacrifices sanglants qui ont lieu dans l'adekpóe, assagis et domestiqués de cette manière, ils insufflent de leur puissance au chasseur et lui portent chance. C'est pourquoi ces enclos sont encore qualifies d'adek lamakpoe (1'enclos de la chance à la chasse, ou l'enclos de l'aklama de la chasse), bien qu'il n'y ait pas lieu d'assimiler tout bonnement ce qu'ils contiennent à un autel ordinaire d'Ak zama.

De même que sont entreposés dans 1'adekpọe des crânes et mâchoires de gros animaux, des crânes et des mâchoires d'ennemis tues au combat (ou de prisonniers sacrifiés), sont entreposés dans le sanctuaire d'un trône de guerre.

Constatons donc que les trônes à cinq pieds sont tous caractéristiques de cultes qui concernent : soit les esprits d'êtres vivants morts de mort violente, ou privés de funérailles traditionnelles; soit les composantes spirituelles de l'home. (Ak Zama jumele à Gbogboo, et tête sous son aspect de tro. la tête étant le principal siège physique du Gbogbol.

Cette constatation effectuée, il y aurait longtemps à épiloguer sur l'étroite association du trône à cinq pieds et des jumeaux. 
Les jumeaux se présentent comme les gardiens de l'antre, ou du bois sacré (coin de forêt primaire où se déroulent les initiations), les êtres de brousse se tenant au goulot d'étranglement qui sépare le monde puissant et dangereux de la source cachée d'énergie vitale (laquelle est caractérisée par le nombre 5 exprimé sur le trône à cinq pieds) du monde phénoménal où l'énergie est attênuée, donc accessible sans rigoureuses précautions (son déployement est caractérisê par le nombre 12 qui sert à diviser l'année ou le zodiaque, ou par un multiple de 12 : 36 ou 60).

L'un des jumeaux oriente vers l'intérieur, vers la source, vers la potentialité toujours jugée mâle par les Evhé (Mawu mâle, forêt primaire mâle).

L'autre jumeau oriente vers l'extérieur, vers l'apparence où la potentialité s'est toute entière transformée en travail, en réalités concrètes... dispersion, démultiplication, passivité terminale, toujours jugée femelle par les Evhé (terre, et terre cuitivée, femelle).

Quant au trône à cing pieds lui-même, il est clair que ses quatre pieds périphériques symbolisent les 4 orients, les 4 Afadu primordiaux, les 4 catégories fondamentales de la pensée géomantique qui correspondent aux quatre éléments".

Le gros pied central (associé au dieu de la foudre xébieso ou Shango) (1) représente le pilier du monde, analogue au pilier de zeus, expression de l'indispensable $5^{\circ}$ élément - insertion de l'absolu dans le relatif - jouant le rôle de moteur dans l'ensemble du monde en devenir, en particulier dans le monde de la vie.

Le socle et le siège du trône représentent la dualité primordiale exprimée par l'en-haut et l'en-bas ; le ciel (rond ou curviligne) et l'abime terrestre (carré, ou plat et anguleux); la lumière et la nature humide ténébreuse; les deux points de vue complémentaires et opposés selon lesquels tout doit être envisagé et qui permettent d'associer à chaque divinité une parèdre.

(I) R. Bastide. Le candomblé de Bahia - rite nago. Paris, Mouton, 1958, pp. 71-72. 
Pourquoi le trône à cinq pieds ? pourquoi cette reprêsentation de la source cachée d'énergie vitale, est-elle particulièrement indiquée pour représenter les entités spirituelles ? Parce que le souffle (gbogbo) joue précisément dans l'organisme vivant le rôle de moteur énergetique (1). Ce même rôle est tenu dans notre cosmos par l'élément Air atmosphérique (auquel le souffle appartient) siège de l'éclair et de la pluie. Et, de même que l'air atmosphérique sépare et unit l'airéther et le feu d'une part, la terre et l'eau d'autre part, le souffle gbogbo susceptible d'errer dans le monde de la vie après la mort sépare et unit le gbogbo qui s'en retourne a Mowu et le tuvho d'une part, le Sè et le corps physique d'autre part.

Enfin, pourquoi le trône à cinq pieds est-il non seulement un autel, mais encore un trône, un siège ?

Nous ne pouvons nous empêcher de mettre ce fait en relation avec la théorie du Yoga, selon laquelle la remontée vers le haut de I'énergie de la Kundalini, qui n'est autre que l'énergie de la parèdre de Shiva, pour aller reconstituer l'Unité transcendantale en s'unissant a l'énergie de shiva, dépend de l'union du souffle au feu de la base (mula agni) lequel est situé dans le centre subtil inférieur, à l'extrémité du sacrum (2).

Ce point de vue admis, l'intronisation, par mise en contact avec le feu de la base du souffle rassemblé sur le trône, est donc en réalité une initiation. Elle est l'initiation par excellence.

(1) Remarquons, au passage, que les Yogi distinguent 5 souffles.

(2) Cf. Yogakundalini upanisad, pp. 7, 45, 74 et 75. 


\begin{tabular}{|c|c|c|c|}
\hline Potentialité & Air-ether & $\begin{array}{l}\text { enveloppe } \\
\text { ethoree du } \\
\text { monde }\end{array}$ & \multirow{2}{*}{$\begin{array}{l}\text { monde } \\
\text { den-haut }\end{array}$} \\
\hline \multirow[t]{2}{*}{ Structure } & Fey & $\begin{array}{l}\text { etage } \\
\text { colesto }\end{array}$ & \\
\hline & $\begin{array}{l}\text { Air } \\
\text { atmosphérique }\end{array}$ & $\begin{array}{l}\text { zône des } \\
\text { perturbations }\end{array}$ & \\
\hline Energie & Eàu & $\begin{array}{l}\text { étage } \\
\text { infernal }\end{array}$ & monde \\
\hline Dévéloppement & Terre & $\begin{array}{l}\text { Plan de } \\
\text { lexistence }\end{array}$ & d'en-bas \\
\hline Dynamisme & Elément & $\begin{array}{l}\text { étage } \\
\text { cósmique }\end{array}$ & \\
\hline
\end{tabular}

\begin{tabular}{|c|c|}
\hline \multirow{2}{*}{$\bar{N} O 1 \mathrm{i}$} & Gbog bo divin \\
\hline & Luvho \\
\hline & Gbogbo vital \\
\hline \multirow{2}{*}{$\begin{array}{l}\text { elements } \\
\text { périssables }\end{array}$} & Sेe \\
\hline & Lâme \\
\hline
\end{tabular}

Hierarchie des constituants del homme dans la tradition Eviné

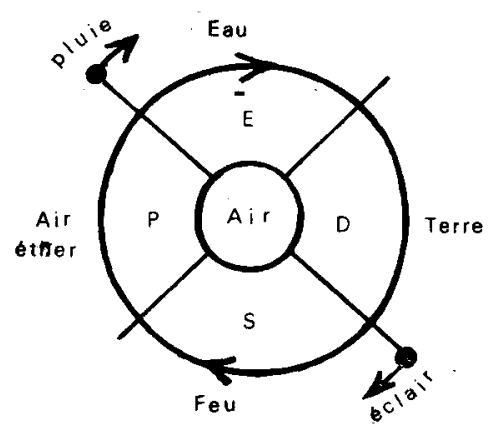

Cycle de transformation des eléments

( chacun des éléments périphériaues étant affecté par l'un des quatre dynamismes fondamentaux )

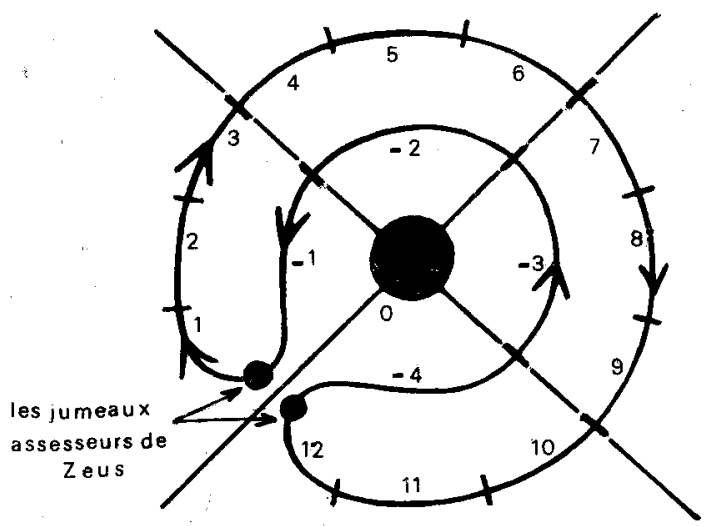

Les 2 I catónories ofomantiguss dy monde en devenif (I) lou les 20 états caractérises de tout systàme en devenir par rapport à un tat initial de référence) :

I élément central (axe immobile de référence) associé à la souverainete du vodu de la foudre

4 éléments (ou Afadu)intégrés à un circuit interne

I2 eléments integrés à un circuit externe 5 eléments du principe vital cache dans $1^{\prime}$ etre vivent

I 12 categories principales de la manifestation

(i) $(16+4+1)=21$ est le chiffre de Leobe, divinite du devenir, de la génération, de le commmication ou de la transgression des categoriw formalles. 


\section{Bibliographie sommaire}

- Agossou J.M. Gbeto et Gbedhoto - L'homme et le dieu créateur sezon les Sud-Dahoméens (Thèse de doctorat en théologie) - Paris, Beauchesne, 1972.

- BASTIDE R. Le candomblé de Bahia - Rite Nago - Paris, Mouton 1958

- GABA C.R. - Anlo traditionnal religion - Ph. D. Thesis London, University of London, $1965-495 \mathrm{pp}$. Illus 10 1/4

- HERSKOVITS M. - Dahomey, an ancient West-African Kingdom New-York, 1938, tome I pp. 194-238 et tome 2 : pp. 231-244

- MAUPoll B. La géomancie d l'ancienne Côte des Esclaves Paris, Institut d'Ethnologie, 1943, 690 pp.

- MONTILUS G. "L'Homme dans la pensée traditionnelie Fon"brochure ronéotypée - Cotonou, 1972

- NENOUNENE J. "Les esprits dans les croyances traditionnelles africaines et dans la bible" - Thèse de théologie Paris, Faculté de Théologie protestante, 1965

- NUKUNYA G.K. "Some underlying beliefs in ancestor worship and mortuary rites among the Ewe" in La notion de personne en Afrique Noire - Paris, Ed. du C.N.R.S., 1973 - pp. 119-130. 